\title{
AUTOMAZIONE CONTABILE E FUTURO DELLA PROFESSIONE CONTABILE
}

\section{ARTICOLO ORIGINALE}

SUMAR, Ramiro Rodrigues ${ }^{1}$

SUMAR, Ramiro Rodrigues. Automazione contabile e futuro della professione contabile. Revista Científica Multidisciplinar Núcleo do Conhecimento. Anno 06, Ed. 06, Vol. 17, pp. 167-181. giugno 2021. ISSN: 2448-0959, Link di accesso: https://www.nucleodoconhecimento.com.br/contabilita/professione-contabile, DOI: 10.32749/nucleodoconhecimento.com.br/contabilita/professione-contabile

\section{RIEPILOGO}

La presenza di qualità nei servizi forniti è infatti indispensabile per la sopravvivenza della professione contabile, in quanto si traduce nell'obiettivo di un commercialista. I sistemi informativi, le nuove tecnologie di intelligenza artificiale e l'innovazione, se collegati alle competenze dei professionisti della contabilità, possono tradursi in maggiori prestazioni delle aziende e, di conseguenza, dell'economia. Considerando che la professione di commercialista cerca di fornire informazioni fiscali al fisco, questo articolo mira a dimostrare che questa professione è in un processo di migrazione per quanto riguarda l'automazione dei compiti, al fine di diventare uno strumento di gestione in grado di assistere nel processo decisionale assertivo, al fine di contribuire allo svolgimento della responsabilità sociale delle imprese. La problematicizzazione di questo studio si basa sulla seguente domanda problematica: quale sarà il ruolo del contabile con l'automazione della contabilità? La professione contabile terminerà come previsto dall'Istituto Sapiens nella ricerca del 2018? Per raggiungere gli obiettivi, è stata utilizzata la rassegna della letteratura. Come risultato, l'articolo ha dimostrato il ruolo del commercialista nel processo di sviluppo e crescita delle aziende prima e dopo

\footnotetext{
${ }^{1}$ Laurea magistrale in Controllo, Specialista in Gestione Tecnologica, Amministrazione Strategica, Sicurezza delle Informazioni e Laureato in Scienze Contabili.
} 
l'automazione dei processi burocratici, nonché la prospettiva dello scenario futuro della professione. Così, si è scoperto che la professione contabile subisce un improvviso cambiamento di posizionamento in modo che sopravviva come professione e mantenga il suo ruolo con la società e l'economia del paese.

Parole chiave: Automazione contabile, Professionista contabile, Responsabilità Sociale, Processo decisionale.

\section{INTRODUZIONE}

C'è uno scenario di grandi cambiamenti nel mercato contabile, con nuove tecnologie, utilizzo dell'intelligenza artificiale e automazione dei processi. E insieme a questi cambiamenti ci sono le preoccupazioni, le ansie e le incertezze che il nuovo suscita nelle persone (SOUZA et al., 2018).

Questi cambiamenti nel paese sono visti e avvertiti da tutti, dove l'inflazione non è più considerata un problema, i tassi di disoccupazione sono diminuiti, l'accesso al credito e ai consumi è aumentato e di conseguenza i tassi di povertà sono diminuiti (CUNHA, 2019).

Esiste quindi uno scenario imprenditoriale, in cui ogni giorno emergono nuove aziende e si richiedono più professionisti, ma alcune professioni corrono il rischio di non essere esistenti a causa della tecnologia e dell'automazione (LEITE, 2017; ARAÚJO, 2020).

Secondo Rozenbaum (2018), uno studio condotto nel 2018 dall'istituto francese Sapiens rivela che cinque professioni sono a rischio di estinzione, tra cui il professionista contabile che lavora in un ufficio senza accesso alla tecnologia, come l'uso di computer.

II contabile moderno deve ancora occuparsi della parte burocratica, delle legalità e di gran parte dei ruoli che occupano spazio. Poiché l'automazione dei processi e altri avvenimenti per facilitare il lavoro contabile derivano dalle tecnologie, il professionista contabile dovrà adattarsi all'uso della tecnologia a suo vantaggio, o essere riluttante a 
cambiare e vedere la sua professione scomparire prima dell'automazione (DINIZ, 2014).

Secondo lo studio dell'istituto francese, più di due milioni di persone "hanno una forte possibilità di veder scomparire il lavoro nei prossimi anni". I dati per lo studio provenivano da DARES (Servizio di studi statistici del Ministero del Lavoro di Francia) e in esso, ad esempio, si può osservare che gli impiegati di banca potrebbero scomparire del tutto entro il 2050 e i contabili entro il 2056 (ROZENBAUM, 2018) .

La competitività esposta sul mercato, con imprenditori alla ricerca di innovazione e nuove tecnologie ogni giorno per espandere il proprio business, rende il professionista contabile inserito in questo ambiente. Le aziende che affrontano il mercato competitivo non possono non avere a fianco dell'aiuto del commercialista, poiché questo professionista ha nel suo obiettivo di attribuzione la qualità e l'efficienza in tutti i settori del business (CHIAVENATO, 2004).

Un altro punto è che i millennials stanno prendendo il controllo del mercato del lavoro e guadagnando posizioni nella gerarchia delle aziende e nel processo decisionale. Quindi per il professionista contabile è importante conoscere e comprendere questo pubblico che si aspetta agilità e qualità nei servizi contabili.

Tuttavia, lo scopo di questo articolo è quello di studiare il futuro della professione contabile e l'automazione che si è verificato nel lavoro contabile. Questi cambiamenti sono necessari per mantenere il contabile nel mercato competitivo, nonché la sua importanza nella creazione di nuovi posti di lavoro, la sua capacità di intraprendere e innovare, nonché l'importanza sociale prima della sua funzione contabile e fiscale.

La professione contabile sta attraversando grandi trasformazioni, non essendo più il professionista che porta solo problemi e conti e fungendo da supporto nella gestione delle aziende. A tal fine, il professionista deve adattarsi alle nuove esigenze e innovare, al fine di mantenere il suo ruolo e la sua importanza, non solo per le imprese e i manager, ma anche per la società, dopo il suo ruolo sociale. 
La metodologia utilizzata nella stesura di questo articolo è stata la ricerca bibliografica, attraverso annais, ricerca, libri, riviste, interviste su Internet, articoli su Internet e articoli accademici pubblicati. Per Gil (2008), la ricerca bibliografica è sviluppata sulla base di materiale già elaborato e composto principalmente da monografie, tesi, articoli scientifici e libri.

Quando si tratta di scopo, Marconi e Lakatos (1992) spiegano che la ricerca bibliografica fa sì che l'osservatore abbia la possibilità di contattare direttamente $\mathrm{i}$ materiali scritti sul tema della ricerca e assistere il ricercatore nella sua analisi o al momento della manipolazione delle informazioni. La ricerca bibliografica è considerata il primo passo verso l'avvio della ricerca scientifica.

\section{RIFERIMENTO TEORICO}

\subsection{STORIA DEL PROFESSIONISTA CONTABILE}

Si ha notizia che la figura del ragioniere sia presente fin dall'inizio della civiltà. Secondo ludícibus e Marion (2006, p. 32): "[...] fin dai popoli più primitivi, la Contabilità esisteva già per la necessità di controllare, misurare e preservare il patrimonio familiare e anche per lo scambio di beni per una maggiore soddisfazione delle persone [...]".

Nonostante i rapporti, l'esistenza ufficiale della contabilità fu segnata nell'età moderna, tra il XV e il XVI secolo, nella regione d'Italia. Nel periodo rinascimentale, la professione contabile acquisì spazio in settori quali: economia, arti, scienze e tecnologie (FIGUEIREDO; STRASSBURG, 2008).

In Brasile, ancor prima che apparisse la prima scuola tecnica commerciale, la contabilità era praticata dalla figura del "contabile", un professionista definito secondo il Codice Commerciale del 1850 che, secondo Francisco D'Auria (1949), si occupava e registrava le transazioni di stabilimenti commerciali in quel momento (CARVALHO, 2017). 
Lo sviluppo della professione contabile ha seguito l'approvazione del decreto-legge 9.295, del 27 maggio 1946, che ha istituito il Consiglio federale dei conti e ha definito le attribuzioni di ragionieri, tecnici contabili e contabili (DOS SANTOS et al., 2019).

Con il consiglio la professione si espanse e sorsero molti problemi con la perequazione di professionisti con diversi livelli di istruzione e conoscenza. Se, da un lato, c'è il commercialista formato con un corso di laurea, un professionista senza alcuno studio coesiste nella stessa professione (NETO, 2010).

Ciò causò il disinteresse dei giovani per la contabilità, poiché lo status di questa professione non era lo stesso di quello di altre formazioni come il diritto e l'economia. Come suscitare interesse per una professione che presenta livelli di professionisti opposti l'uno all'altro? Ciò è stato possibile solo con la prova di competenza e separazione dal tecnico contabile al contabile stesso.

Nel 1972, l'audit fu regolato dalla risoluzione n. 220, così come dalle circolari n. 178 e n. 179 della Banca Centrale del Brasile. Ha imposto standard dimostrativi che richiedevano che i professionisti siano adattati a un audit da parte di persone certificate (ABREU, 2013; CORDEIRO, 2011).

Tuttavia, la professione contabile ha ricevuto lo status solo nel dicembre 1976 con l'emanazione della legge 6.404 nota come legge SA (Sociedades Anônimas - Società anonima). Questa legge ha dato origine alla Brazilian Securities and Exchange Commission (CVM), che ha sostituito la Banca centrale, per quanto riguarda l'emissione di pareri e parte della legislazione contabile, nonché il controllo del mercato dei capitali in Brasile (CARVALHOSA, 2017).

Per Santos (2008, p. 9)

[...] negli ultimi anni, soprattutto dopo la fine dell'era dell'alta inflazione, a partire dalla metà degli anni ' 90 , ha attraversato un periodo di grandi cambiamenti e adattamenti. Le aziende che sono state più agili nell'adattare la loro attenzione al loro core business, al loro core business e nel non gestire più gli utili e i ricavi inflazionistici, hanno raggiunto un tasso di successo più elevato. $\mathrm{E}$, in questo momento, il professionista contabile ha una partecipazione primaria quando 
fornisce orientamento fiscale, societario e finanziario. [...]. II bancone, sempre più spesso, è la bussola dell'azienda.

Si osserva che le competenze e le competenze del professionista contabile sono focalizzate sulle strategie e sul processo decisionale nelle aziende. A causa dei cambiamenti tecnologici, governi, aziende e manager sono in un alto grado di innovazione, sempre più esigenti da parte del professionista contabile che, a sua volta, assiste il processo decisionale (DE OLIVEIRA REIS et al., 2015).

In un ambiente altamente competitivo e agile, ottenere informazioni in sicurezza è un differenziale. Precisione e affidabilità sono richieste anche dalle aziende moderne che vogliono garantire la loro sopravvivenza sul mercato e rendere la figura contabile un data scientist patrimoniale e voci contabili, richiedendogli di sapere come interpretare i dati e delineare strategie e percorsi che garantiscano risultati migliori. Tutte queste richieste si contronotono a ciò che Santos $(2008$, p. 10) scrive nel suo libro:

[...] il contatore non dovrebbe perdere di vista il fatto che la contabilità non è per questo, è per l'utente. La contabilità è il linguaggio degli affari e questo linguaggio racconta la storia di ogni azienda. Perfezionare questo linguaggio cercando di aumentare il suo potere di previsione e utilità per l'utente è forse la missione principale del contabile. [...]

Pertanto, il professionista deve essere in fase di apprendimento, aggiornamento e formazione per essere un esperto nel suo ruolo e nell'ottenere dati che aiutino l'azienda nel suo obiettivo sociale. II facile accesso ai corsi post-laurea nel settore della contabilità offre all'individuo l'opportunità di essere posizionato accanto al mercato per contribuire alle imprese, all'economia e alla società.

\subsection{SVILUPPO ECONOMICO E CRESCITA}

Furtado (1964) definisce lo sviluppo economico come "un processo di cambiamento sociale attraverso il quale un numero crescente di bisogni umani è soddisfatto attraverso una differenziazione del sistema produttivo derivante dall'introduzione di innovazioni tecnologiche". 
Per Hewlett (1981), lo sviluppo economico è definito come "un aumento significativo del reddito reale pro capite di una nazione, allo scopo di ottenere cibo, salute, istruzione, migliori condizioni di vita e una gamma sempre più ampia di opportunità di lavoro e di svago per la popolazione di questa nazione".

D'altra parte, c'è una crescita economica che, secondo Siedenberg (2006), consiste in un "processo di cambiamenti di carattere prevalentemente quantitativo, che significa un aumento di dimensioni, volume e / o quantità". Riassumendo ciò che Vasconcellos (2000) ha concluso, sottolineando che la crescita economica è la continua crescita del reddito pro capite nel tempo.

La crescita e il mantenimento del prodotto interno lordo (PIL) promuovono una migliore qualità della vita della popolazione. Inoltre, le innovazioni tecnologiche, l'aumento delle entrate nazionali, l'espansione della forza lavoro e il risparmio sono altri fattori che aiutano nella crescita economica di un paese.

Si osserva che quando un paese mostra una crescita economica il numero di disoccupati diminuisce, mentre le imposte riscosse aumentano. In questo contesto, è necessario investire di più per mantenere la crescita continua dell'economia. Mendes (2015) spiega che l'economia e la crescita dei genitori hanno sottoperformato a causa di alcuni fattori, tra cui l'istruzione e l'elevato carico fiscale.

In linea con la crescita economica arriva lo sviluppo economico che per esistere è necessario che il paese mantenga indicatori e abbia fattori per migliorare la qualità della vita e il reddito della popolazione nel tempo. Per Rocha (2004) ciò che dimostra questi fattori è: la crescita del benessere economico, misurata attraverso indicatori economici, tra i quali vale la pena menzionare il prodotto nazionale totale e il prodotto nazionale pro capite; ridurre i livelli di povertà, disoccupazione e vita sociale; e l'aumento delle condizioni di salute, nutrizione, istruzione e alloggio.

L'Indice di Sviluppo Umano - ISU, delle Nazioni Unite (ONU), è un'unità di misura creata e utilizzata per verificare il grado di sviluppo di un determinato Paese, che copre salute, istruzione e reddito. L'indice è un riferimento numerico la cui variazione è 
definita tra 0 e 1 , nelle sue definizioni, più il valore è vicino a 1 , migliore dovrebbe essere lo sviluppo di quella posizione negli elementi presentati. Nessun paese ha un ISU definito pari a zero o addirittura uno (FILGUEIRA et al., 2010).

Secondo il rapporto delle Nazioni Unite che ha la valutazione di 189 paesi, il Brasile ha raggiunto 0,761 punti nel 2018 , che rappresenta un aumento significativo rispetto al 2013, dove ha ricevuto 0,752 .

Il Brasile è al $79^{\circ}$ posto della classifica, insieme alla Colombia e si colloca tra i paesi nella categoria ad alto sviluppo umano. Nel rapporto, l'indicatore più alto è la Norvegia con 0,954 punti e elencato con il peggior indice di sviluppo umano è il Niger con 0,377 (LEUSIN JÚNIOR, 2015).

\subsection{NUOVE TECNOLOGIE IN CONTABILITÀ}

Le nuove tecnologie finiscono per generare cambiamenti strutturali nelle organizzazioni con la loro applicazione, imprendo sui costi delle aziende e modificando i loro processi produttivi, in considerazione della competitività sul mercato (MAT, 2010).

Scott (2009) sottolinea che l'uso della tecnologia nella contabilità è un cambiamento senza precedenti, cioè non è facile identificare se i suoi impatti fossero negativi o positivi. Da un altro punto di vista, Alsharayri (2011) e Choe (2004) mostrano che il livello di tecnologia e informazione prodotta ha impatti positivi.

La tecnologia ha impatti positivi sia sull'agilità che sull'affidabilità e sulla sicurezza dei sistemi informativi utilizzati nelle aziende da uffici contabili e professionisti. La funzione delle informazioni generate dai sistemi, secondo Allahyari e Ramazani (2011), è quella di aiutare i propri utenti a prendere decisioni, considerando che la contabilità è in grado di produrre informazioni rilevanti per la formulazione di processi aziendali e strategici, per il controllo delle attività e per la crescita delle aziende.

Per Scott (2009), il vantaggio della tecnologia sta nell'uso di diversi strumenti per accelerare l'esecuzione delle funzioni eseguite dal contatore. In questo aspetto, la 
tecnologia contribuisce al progresso operativo della contabilità di fronte alla competitività, data la velocità e l'efficienza offerte dai progressi tecnologici.

Acevedo (2012) mostra che se le comunicazioni nelle società di contabilità sono veloci possono contribuire ad aumentare la produttività, oltre a migliorare il processo decisionale e facilitare la crescita aziendale.

Sempre secondo Scott (2009), uno svantaggio della tecnologia è la sua dipendenza dagli esseri umani. La tecnologia nasce dall'innovazione umana, quindi, è un prodotto che ripete gli stessi errori umani. Ma questo può essere aggirato con processi più robusti e con una specializzazione e un'educazione più mirate sull'automazione. Dimostra anche che un altro svantaggio è la dipendenza della professione dalla tecnologia, ma è già noto che gli studi dimostrano che il contatore che esisteva per rendere operativi i calcoli si estinguerà, il che si contrae da questo pensiero errato dell'autore.

Aribaba et al. (2011), a loro volta, evidenziano l'importanza che la tecnologia aggiunge alla società, soprattutto nelle piccole società di fornitura di servizi, dato il miglioramento delle prestazioni e il maggiore sviluppo del business. Pertanto, con maggiori prestazioni, sorgono più opportunità e guadagni, con ciò vengono generati posti di lavoro e l'economia ne beneficia.

La competitività richiede alle organizzazioni contabili di essere in grado di creare valore per i propri clienti. Clienti che sono contribuenti e appaltatori del personale. Secondo Simons (1987), differenziandosi dai concorrenti, implementando e formulando una strategia aziendale ben definita, queste organizzazioni aggiungeranno più risultati. Nella strategia, l'uso di tecnologie aggiunte ai sistemi contabili è essenziale, in quanto fornisce informazioni sicure e utili (JERMIAS; GANI; 2004).

Avere una strategia ben definita e consolidata, basata su investimenti in tecnologia e qualificazione delle persone, secondo Grande et al. (2011), porterà vantaggi produttivi e cambiamenti favorevoli ai suoi clienti rispetto ai suoi concorrenti. 


\subsection{RUOLO DEL CONTABILE NELL'INNOVAZIONE E NELL'AUTOMAZIONE DEI PROCESSI CONTABILI}

II numero degli uffici contabili aumenta e contribuisce alla diminuzione della disoccupazione tra il 2009 e il 2012, secondo Gondim (2014). C'è stato un aumento di oltre il $60 \%$ nei lavori formali e del $5 \%$ nelle nuove società di contabilità.

II Consiglio federale dei conti (CFC, 2014) espone, sul proprio sito web, un sondaggio realizzato che dimostra che oltre il $58 \%$ dei professionisti della classe contabile preferisce lavorare in aziende private $\mathrm{o}$ aprire un proprio studio piuttosto che dover sostenere l'esame ed entrare nel settore pubblico. Vedi che questi dati mostrano il contributo alla generazione di numeri di posti di lavoro formali e informali, poiché il professionista contabile lavora con i dirigenti delle aziende (i loro clienti) collaborando in modo che l'azienda si evolva e cresca e abbia bisogno di più dipendenti.

Aiutando gli imprenditori o investendo nella propria attività, utilizzando le conoscenze pratiche e teoriche acquisite, il ragioniere ha un ruolo significativo nell'economia. Negli ultimi anni, la contabilità ha smesso di utilizzare solo una calcolatrice e risme di carta (NOGUEIRA, 2010).

Si veda che Coelho (2015) spiega che il commercialista è passato dall'essere la persona che registra fatti passati ad essere un consulente strategico con una visione prospettica e ampia del mercato. Sulla base di questi cambiamenti il contactor ha la missione di automatizzare i processi, generando più prestazioni e comcomworking dati statistici al fine di supportare aziende e manager nel processo decisionale.

Favero et al. (2006) mostrano che "la contabilità mira a generare informazioni per diversi utenti in modo che possano prendere decisioni". Questo obiettivo sembra semplice, ma i fattori soggettivi in esso coinvolti rendono difficile questo processo, la difficoltà di comprensione dei dati e la distanza tra il contabile e il manager rendono difficile il raggiungimento di questo obiettivo. Vediamo che ci sono variabili per una buona comunicazione, e queste devono essere studiate e la specialità del ragioniere, in modo che il tuo obiettivo, come professionista della contabilità, sia raggiunto. 
Quale target di riferimento per le informazioni contabili, viene individuata la nuova generazione che necessita di informazioni affidabili e richiede che sia fatta rapidamente, d'accordo con Drucker (1987), il compito specifico del manager imprenditoriale di un'azienda è quello di rendere il business di questa azienda per diventare più agile e di qualità.

Secondo i dati disponibili sul sito web CFC (2014), il Brasile ha attualmente più di 71.008 organizzazioni/società contabili e 515.011 professionisti registrati presso il Consiglio federale dei conti e questi professionisti e uffici devono essere preparati per automatizzare i processi.

Avendo il commercialista la funzione di guidare i manager alla crescita delle loro aziende, questo professionista svolge anche un ruolo importante nella società, e per questo, dovrebbe sempre essere alla ricerca di aggiornare le proprie conoscenze e comportarsi eticamente, essendo l'interlocutore del governo, con il contribuente e la società (CORONADO, 2017).

II commercialista che guida i suoi clienti a contribuire correttamente, aiuta nello sviluppo dello stato e con questo nello sviluppo del paese. In questo ruolo, l'automazione è la chiave per tutta l'attenzione del contabile è nella generazione di informazioni e non in attività noiose e dispendiose in termini di tempo di conferenze e rilasci.

Competenza, professionalità, agilità e specializzazione di chi è coinvolto nei processi li rendono più coerenti, agili e funzionano correttamente. Una pratica contabile efficiente e specializzata è il punto che dovrebbe essere prestato attenzione al perpetuo business e sono sempre più efficienti e competitivi.

II professionista che comprende questi cambiamenti comportamentali che sono accadsi, è specializzato e aggiorna, avrà un ruolo importante e decisivo nella società e nelle aziende. L'intero processo dipende da un essere umano, e questo, l'essere umano deve avere le competenze e le competenze affinché il processo automatizzato funzioni correttamente, oltre a una conoscenza sistemica delle aziende. 
Specializzazione e conoscenza approfondita sono strumenti per questo nuovo paradigma di cambiamento nella professione contabile.

\section{CONCLUSIONE}

Come affermato in questo articolo, la professione contabile fin dall'inizio è importante per lo sviluppo e la crescita del paese. L'automazione dei processi contabili e il cambiamento dei requisiti della professione faranno estinguere il professionista che oggi è visto solo come il rispetto dei requisiti di legge. Poi arriva l'opportunità per la professione e i professionisti di distinguersi in altre aree delle loro conoscenze come la consulenza e la strategia aziendale, mettendo le vaste conoscenze acquisite nell'istruzione per la formazione.

Gli uffici contabili contribuiscono alla creazione di posti di lavoro, svolgendo così il loro ruolo sociale, ma devono adattarsi alle nuove dinamiche dei sistemi informativi in modo che siano efficienti e producano con qualità e agilità le informazioni necessarie per le imprese e i governi. Poiché l'istruzione è una base per lo sviluppo di qualsiasi nazione, il miglioramento della professione e dellistruzione del professionista comporta un significativo miglioramento della base della società e con essa eventi di miglioramento sociale e professionale.

Il professionista contabile ha cessato di essere quello che porta solo cattive notizie alle aziende, con guide e contabilità da pagare, fornendo un orientamento strategico a imprenditori e manager, contribuendo a svolgere le loro funzioni correttamente e con l'agilità che il mercato richiede attraverso dati corretti ed estratti rapidamente.

Contador ha tutti gli strumenti e le conoscenze per automatizzare la preparazione dei bilanci e delle uscite, al fine di portare più agilità e affidabilità, emerge così come alleato per le aziende per svolgere il proprio ruolo sociale con la crescita del business.

Le aziende che crescono in modo solido, intelligente e adempiono ai loro obblighi fiscali e societari con qualità e agilità, un ruolo che il commercialista può svolgere insieme al management, faranno sviluppare l'intera società. Se tutti adempiranno al 
loro ruolo con l'economia e la società, si generano nuovi posti di lavoro, si pagano più tasse, si diminuiranno i tassi di povertà e l'offerta di istruzione sarà più elevata.

La minaccia dell'inesistenza della professione farà sì che i professionisti si reinventino e rivedano la loro posizione di fronte alle innovazioni. Chi ha una visione diversa della propria posizione di professionista contabile, coniugarsi con le nuove tecnologie di intelligenza artificiale, automazione e specializzazione ha guadagnato spazio sul mercato, diverso dal professionista ignaro dei cambiamenti e della lotta contro di loro.

D'altro canto, l'educazione contabile deve essere migliorata, in modo che le nuove tecnologie siano utilizzate nell'insegnamento e che i tirocinanti lascino le università disposte ad affrontare il mondo attuale che richiede agilità e qualità nella fornitura di servizi.

Si osserva un divario da studiare per quanto riguarda la distribuzione demografica dei professionisti della contabilità, dato il grado di innovazione delle imprese in alcune regioni. Ciò darà una visione più ampia di ciò che può accadere o meno con la carriera. Si suggerisce inoltre di condurre studi di casi in aziende innovative con un alto grado di automazione e il posizionamento del professionista nei confronti di questa azienda e dei suoi risultati. Questi studi contribuiranno a una più ampia comprensione di ciò che potrebbe accadere in futuro.

\section{RIFERIMENTI}

ABREU, E. N. de. Regulamentação de auditoria: estudo da influência das fraudes contábeis de 2001. Goiânia: Universidade Federal de Goiás - UFG, 2013.

ACEVEDO, L. Business benefits of information technology. Chron, 2019. Disponível em: <http://smallbusiness.chron.com/business-benefits-informationtechnology-4021.html . Acesso em: 10/mai/2021

ALLAHYARI, A.; RAMAZANI, M. Firm technological change and its effects on management accounting change: case study of Iranian manufacturing firms. Global Journal of Management and Business Research, v. 11, n. 9, 2011. 
ALSHARAYRI, M. A. The E-Commerce impact on improving accounting information system in Jordanian Hotels. International Research Journal of Finance and Economics, v. 75, 2011.

ARAÚJO, F. M. de. A inteligência artificial e os seus impactos no mundo do trabalho. Fortaleza: Centro Universitário Fametro, 2020.

ARIBABA, F. et al. An evaluation of the impact of technological innovative entrepreneurial development programmes on the performance of small scale business in Nigeria. Global Journal of Business, Management And Accounting, v. 1, n. 1, 2011.

CARVALHOSA, M. de S. B. Comentários à lei de sociedades anônimas. Saraiva Educação SA, Editora: Saraiva Educação S. A., v. 1. 2017.

CFC. Conselho Federal de Contabilidade. Dados estatísticos do Conselho Federal de Contabilidade. 2014. Disponível em: http://www.cfc.org.br/. Acesso em: 12/mai/2021.

CHIAVENATO, I. Empreendedorismo: dando asas ao espírito empreendedor. Editora manole, $4^{\mathrm{a}}$ ed. 2004.

$\mathrm{CHOE}, \mathrm{J}$. Impact of management accounting information and AMT on organizational performance. Journal of Information Technology, v. 19, 2004.

COELHO, J. M. A. Contabilidade: uma carreira em transformação. Disponível em: http://www.portalcfc.org.br/noticia.php?new=23196. Acesso em: 12/mai/2021.

CORDEIRO, C. M. R. Auditoria e Governança corporativa. Editora: IESDE, 2011.

CORONADO, O. Contabilidade gerencial básica. Editora: Saraiva Educação SA, 2017.

CUNHA, E. S. Oferta de crédito e emprego local nos municípios da região Nordeste: 2002-2015. Salvador: Federal da Bahia. 2019. 
D'AURIA, F. Primeiros princípios de contabilidade pura. São Paulo: Departamento de Cultura e Ação Social, 1949.

DE OLIVEIRA REIS, A. et al. Perfil do profissional contábil: habilidades, competências e imagem simbólica. Revista Contemporânea de contabilidade, v. 12, n. 25, p. 95-116, 2015.

DINIZ, E. R. da S. et al. Governança eletrônica no Brasil e o papel das tecnologias da informação e comunicação. Florianópolis: Universidade Federal de Santa Catarina, 2014.

DOS SANTOS, M. J. A. et al. Um estudo comparativo entre o exame de suficiência do conselho federal de contabilidade, o exame nacional de desempenho de estudantes e a pós-graduação stricto sensu dos cursos de ciências contábeis do Estado do Rio de Janeiro. Polêm!ca, v. 13, n. 4, p. 1681-1698, 2014.

DRUCKER, P. F. Inovação e Espírito Empreendedor: prática e princípios. São Paulo: Pioneira, 1987.

FAVERO, H. L. et al. Teoria e Prática. 4 ed. Editora: Atlas, São Paulo. 2006.

FIGUEIREDO, C. R.; STRASSBURG, U. A Contabilidade: aspectos históricos que influenciaram no seu desenvolvimento no decorrer do tempo. IX Jornada de Estudos Contábeis-UNIOESTE, 2008.

FILGUEIRA, J. M. et al. Um panorama estatístico de cunho econômico, social e educacional do estado do Rio Grande do Norte. HOLOS, v. 4, p. 198-215, 2010.

FURTADO, C. Dialética do Desenvolvimento. Rio de Janeiro, $2^{\underline{a}}$ ed., Fundo de Cultura, 1964.

GIL, A. C. Métodos e técnicas de pesquisa social. 6. ed. São Paulo: Atlas, 2008.

GONDIM, A. MEI também tem obrigações fiscais. Contábeis, 2016. Disponível em: https://www.contabeis.com.br/noticias/26714/mei-tambem-tem-obrigacoes-fiscais/. 
GRANDE, E. U. et al. The impact of accounting information systems (AIS) on performance measures: empirical evidence in Spanish SMEs. The International Journal of Digital Accounting Research, v. 11, 2011.

HEWLETT, S. A. Dilemas do desenvolvimento. Rio de Janeiro: Zahar, 1981.

IUDÍCIBUS, S. de; MARION, J. C. Introdução à teoria da contabilidade para o nível de graduação. 4 ed. São Paulo. Editora: Atlas, 2006.

JERMIAS, J.; GANI, L. Integrating business strategy, organizational configurations and management accounting systems with business unit effectiveness: a fitness landscape approach. Management Accounting Research, v. 15, 2004.

LEITE, E. F. O fenômeno do empreendedorismo. Saraiva Educação SA, 2017.

LEUSIN JÚNIOR, S. A inserção internacional de potências médias: evidências da afirmação de países em desenvolvimento a luz da evolução do sistema multilateral de comércio. Porto Alegre: Universidade Federal do Rio Grande do Sul, 2015.

MARCONI, M. de A.; LAKATOS, E. M. Metodologia do trabalho científico. São Paulo: Editora Atlas, 1992. 4a ed.

MAT, T. Z. Management accounting and organizational change: impact of alignment of management accounting system, structure and strategy on performance. Philosophy School of Accounting, Finance and Economics Faculty of Business and Law Edith Cowan University. Perth Western Austrália, 2010.

MENDES, M. Por que a economia do Brasil foi para o buraco? Brasil economia e governo, 2015. Disponível em: http://www.brasil-economiagoverno.org.br/2015/08/25/por-que-a-economia-brasileira-foi-para-o-buraco/. Acesso em: 14/mai/2021. 
NETO, O. R. M. A profissionalização do contador no Brasil. São Paulo: Mackpesquisa, 2010. http://168.197.92.160/bitstream/handle/10899/14605/721_2008_0_15.pdf?sequence $=1$ \&isAllowed=y/. Acesso em: 14/mai/2021.

NOGUEIRA, M. Práticas de contabilidade: relatório de estágio do Mestrado em Gestão. Coimbra: [s.n], 2010.

RAMALHO, T. C. L. Contabilidade criativa: a percepção dos Técnicos Oficiais de Contas. Lisboa: Instituto Superior de Contabilidade e Administração de Lisboa. 2015.

ROCHA, F. J. M. Educação e economia: uma abordagem sobre as consequências e condicionantes econômicas do desenvolvimento humano, com ênfase em educação. Brasília: Cadastro de Finanças Públicas, n. 5, 2004.

ROZENBAUM, S. Revolução digital deixa cinco profissões à beira da extinção. rfi, 2018. Disponível em: https://www.rfi.fr/br/economia/20180829-revolucao-digitaldeixa-cinco-profissoes-beira-da-extincao-diz-estudo.

SANTOS, R. F. dos. Introdução à contabilidade: noções fundamentais. São Paulo: Saraiva, 2008.

$\mathrm{SCOTT}, \mathrm{W}$. The impact technology is having on the accounting profession. World conference on higher education, 2009.

SIEDENBERG, D. R. Dicionário do Desenvolvimento Regional. Santa Cruz: Edunisc, 2006.

SIMONS, R. Accounting control systems and business strategy: an empirical analysis. Accounting, Organizations and Society, v. 12,1987.

SOUZA, E. C. et al. As características qualitativas da informação contábil a partir da percepção dos profissionais de contabilidade. In: Congresso UFPE de Ciências Contábeis. 2018. 
VASCONCELLOS, M. A. S. de. Economia Micro e Macro: Teoria e Exercícios, Glossário com 260 Principais Conceitos Econômicos. São Paulo: Atlas, 2000.

Inviato: Agosto 2020.

Approvato: Giugno 2021. 